\title{
The Practice of Intormatics
}

Application of Information Technology

\section{Rapid Identification of Hospitalized Patients at High Risk for MRSA Carriage}

\author{
R. Scott Evans, MS, PhD, Carrie Jane Wallace, RN, PhD, James F. Lloyd, BS, \\ Caroline W. Taylor, MSN, Rouett H. Abouzelof, MSN, Sharon Sumner, BSN, \\ Kyle V. Johnson, BS, Amyanne Wuthrich, MS, Stephan Harbarth, MD, MS, \\ Matthew H. Samore, MD, for the CDC Prevention Epicenter Program
}

\begin{abstract}
A b S t r a c t Patients who are asymptomatic carriers of methicillin-resistant Staphylococcus aureus (MRSA) are major reservoirs for transmission of MRSA to other patients. Medical personnel are usually not aware when these high-risk patients are hospitalized. We developed and tested an enterprise-wide electronic surveillance system to identify patients at high risk for MRSA carriage at hospital admission and during hospitalization. During a two-month study, nasal swabs from 153 high-risk patients were tested for MRSA carriage using polymerase chain reaction (PCR) of which 31 (20.3\%) were positive compared to 12 of $293(4.1 \%$, p < 0.001) lowrisk patients. The mean interval from admission to availability of PCR test results was 19.2 hours. Computer alerts for patients at high-risk of MRSA carriage were found to be reliable, timely and offer the potential to replace testing all patients. Previous MRSA colonization was the best predictor but other risk factors were needed to increase the sensitivity of the algorithm.
\end{abstract}

- J Am Med Inform Assoc. 2008;15:506-512. DOI 10.1197/jamia.M2721.

\section{Introduction}

Methicillin-resistant Staphylococcus aureus (MRSA) has become a major public health problem worldwide. ${ }^{1-3}$ Within U.S. hospitals, nearly $60 \%$ of nosocomial Staphylococcus aureus infections acquired in intensive care units are methicillin resistant. ${ }^{4}$ Staphylococcus aureus, whether methicillinresistant or methicillin-susceptible, exhibits a propensity to asymptomatically colonize human hosts. Common anatomic locations of asymptomatic MRSA carriage include the nares, groin, and sites where skin integrity has been breached, such as wounds. Health care workers may carry MRSA on their hands or clothes following contact either with asymptomatic carriers or patients who have clinical infection. Health

Affiliations of the authors: Department of Medical Informatics, LDS Hospital, Intermountain Healthcare (RSE, CJW, JFL, KVJ), Salt Lake City, UT; Department of Biomedical Informatics, University of Utah School of Medicine (RSE, MHS), Salt Lake City, UT; Division of Infection Control, Intermountain Healthcare (CWT, RHA, SS), Salt Lake City, UT; Division of Epidemiology, University of Utah School of Medicine (AW, MHS), Salt Lake City, UT; Department of Internal Medicine, University Hospitals of Geneva (SH), Geneva, Switzerland; VA Health Care System (MHS), Salt Lake City, Utah.

Funding for this study was provided by Grant 1 U01 CI000334-01 from the Center for Disease Control and Prevention. We also thank Becton Dickinson for providing the PCR kits used to test for MRSA carriage.

Correspondence: R. Scott Evans, M.S., Ph.D., FACMI, Department of Medical Informatics, LDS Hospital, $8^{\text {th }}$ Avenue and C Street, Salt Lake City, Utah; e-mail: <ldsevans@ihc.com>.

Received for review: 01/14/08; accepted for publication: 04/10/08 workers may then unknowingly transmit the organism to other patients. Contaminated environmental surfaces also contribute to MRSA transmission. Thus, symptomatic patients constitute a small portion of the actual reservoir of MRSA within hospitals resulting in an iceberg phenomenon. ${ }^{5}$ Reducing patient-to-patient MRSA spread is currently considered the cornerstone for prevention of healthcareacquired MRSA infection.

\section{Background}

The infection control community is divided on the most appropriate strategy to control MRSA transmission among hospitalized patients. ${ }^{6-8}$ MRSA carriers are typically not recognized unless surveillance cultures of sites of colonization are performed. In 2003, a Society for Healthcare Epidemiology Association (SHEA) taskforce advocated a strategy of using active surveillance cultures to identify patients who carried MRSA or vancomycin resistant enterococci (VRE). ${ }^{9}$ It was also recommended that all asymptomatic carriers be placed on contact isolation comprised of the use of private rooms as well as gowns and gloves for all patient contact. However, this strategy incurs substantial financial and physical resource costs for the hospital. Another limitation is the concern that contact precautions may decrease providerpatient interaction and result in increased rates of other adverse events. ${ }^{10,11}$ Simple factors that predict MRSA carriage at the time of hospital admission have been identified. ${ }^{12,13}$ Thus, the use of a prediction rule to detect patients at high risk of MRSA carriage offers a more targeted surveillance strategy. 
Evidence-based strategies to reduce the spread of resistant organisms across the healthcare continuum need to be developed, evaluated, and disseminated. Core requirements for implementation of effective and efficient infection control strategies include timely, bidirectional transfer of relevant information between infection control personnel and diverse types of clinical personnel. Relevant information includes patient risk data and actionable recommendations to support a standardized infection control process. In the case of MRSA, healthcare personnel may be unaware of patients who have had previous MRSA infection at their hospital or another facility. Advances in the use of electronic health records, knowledge bases, and inference engines enable the implementation of such strategies. Moreover, the value of using computerized decision support to help infection control practitioners track encounters by these high-risk patients over a large geographical area has not been studied.

LDS Hospital, the facility where the MRSA alerting system was implemented, is operated by Intermountain Healthcare, an integrated delivery system located in Utah and Southern Idaho. Intermountain Healthcare comprises 21 hospitals, 25 health centers, 92 affiliated outpatient clinics, five dialysis centers, and a home health care service. LDS Hospital has been using an electronic health record (EHR) known as the HELP System for more than 30 years. ${ }^{14}$ Intermountain Healthcare has been developing an enterprise-wide EHR (HELP2) system for more than ten years. ${ }^{15}$ Together the HELP and HELP2 systems provided the electronic infrastructure for determining patient risk. A central feature of the infrastructure is a unique medical number assigned to all patients seen at any Intermountain Healthcare facility during the first encounter and used for unique patient identification in subsequent encounters within the system. Each night all data from the HELP and HELP2 Systems are stored into an enterprise data warehouse (EDW).

\section{Prior Use of Alerts}

Electronic notification of infection control practitioners (ICPs) whenever MRSA was identified on new microbiology test results has been used at LDS Hospital since $1984 .{ }^{16}$ This notification program has been disseminated to the other Intermountain Healthcare hospitals, however it does not notify ICPs when previously identified MRSA patients were readmitted to either the same or a different Intermountain Healthcare facility. A few studies report automated notification of infection control personnel when readmission after discharge from the same hospital occurs, by using stored microbiology results acquired through automated or manual data entry. ${ }^{17-19}$ However, none of these systems use microbiology results from other hospitals or outpatient settings to identify patients with previous positive MRSA results and bedside caregivers are not always notified upon hospital admission.

In an earlier study, we found that patients with previous MRSA from just LDS Hospital had subsequent encounters at 62 different Intermountain Healthcare facilities up to 304 miles away from 1 day to more than 5 years later. ${ }^{20}$ More recently, we expanded the MRSA alerting system at LDS Hospital to notify infection control practitioners when patients with MRSA previously detected at any Intermountain Healthcare facility were admitted. Having an enterprise- wide system for driving infection control alerts provided the basis for the new program described in this paper. The current study was also facilitated by our recent work to implement alerts for critical ventilator events. The ventilator alerting system demonstrated the value of real time communication of the event type and room location on flashing computer terminal screens. ${ }^{21}$

\section{Design Objectives}

Our objective for this project was to test a computer-based alerting system developed to facilitate rapid, targeted surveillance of adult inpatients for MRSA carriage at admission and during hospitalization. We automated an MRSA risk stratification algorithm and computer-based alerting system to notify nurses and infection control practitioners when high-risk patients needed to be tested for MRSA carriage. The alert served as a standing order to obtain a nasal swab for rapid polymerase chain reaction (PCR) testing to document MRSA carriage status. Specific goals were to validate the MRSA risk stratification algorithm and evaluate the alert system usability. PCR was selected over microbiology cultures because results could be available within 24 hours of specimen collection.

\section{System Description}

\section{System-wide MRSA Database}

We developed a system (Figure 1) to evaluate risk of MRSA carriage and alert bedside nurses and infection control personnel when high-risk patients were identified. We developed four similar PLSQL programs to search the EDW and extract all MRSA positive microbiology culture results, patient registration information at all facilities during the previous six months, antibiotics (cephalosporins, fluoroquinolones, carbapenems) ordered or administered during the previous six months, and current dialysis diagnosis. The data were stored on our network in a new MRSA High Risk database. The primary key for all the MRSA High Risk tables was the unique medical number used for patient identification across the system. An automated (time driven) process was used to update the data in the MRSA High Risk tables each night and retain only the most current data.

\section{Identification of High-risk Patients}

The criteria used to identify high-risk patients were adapted from a large-scale validation study ${ }^{22}$ (Table 1). Evaluation of the database to determine risk of MRSA carriage was triggered by either a data- or time-driven process. The HELP system data-driver was set up to activate a medical logic module each time hospital personnel entered admission or transfer (room change) data. The medical logic module verified the room change data and used a "SQL Write" routine to add a row to the "room change" table in the database (unique medical number, room number, admitting facility code, hospital account number, admission/room change date, name, age). Data storage into the room change table activated another PLSQL program to evaluate the risk criteria. Another time-driven program was triggered at 17:30 each day and calculated the length of stay for all current hospital patients and used the high-risk criteria to identify any new high-risk patients. Thus, a risk evaluation occurred for all patients at admission, transfer within the hospital, or extended (10 and 30 day) lengths of stay. Low risk patients 


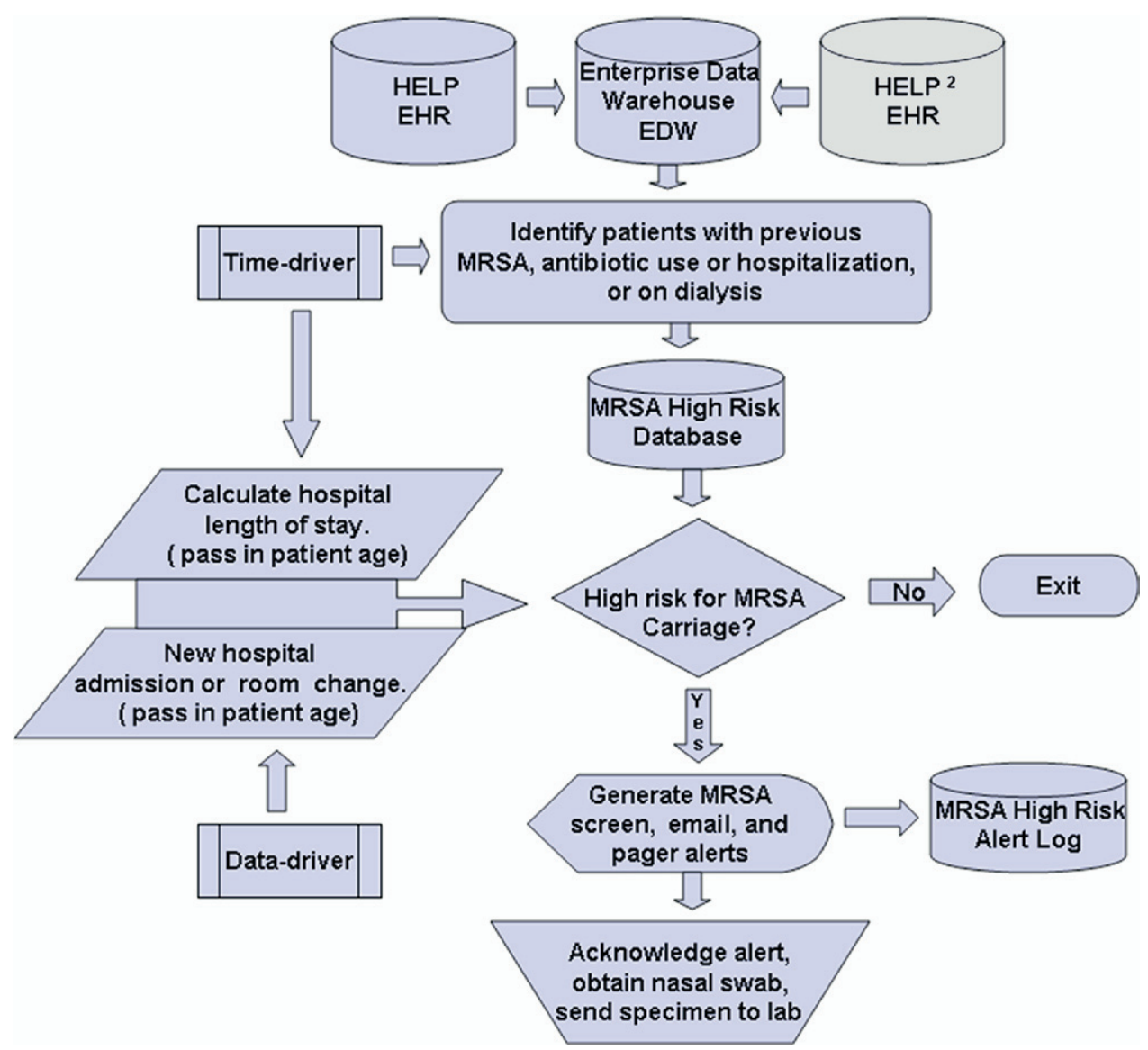

F i gure 1. System description for the generation and acknowledgement of alerts for patients at high risk of having MRSA carriage. $\mathrm{EHR}=$ electronic health record; $\mathrm{EDW}=$ enterprise data warehouse; MRSA $=$ methicillin-resistant Staphylococcus aureus.

comprised the set of all patients stored in the room change table and not identified as high-risk.

\section{High-risk Alert Deployment}

When a high-risk patient was identified, the criteria were stored into a "High Risk Alert" log along with patient identification data, hospital room number and a dichotomous "alert sent" field, which was initially set to 0 . Every 5 minutes, the Java runtime environment activated a Java program to check the High Risk Alert table for rows where the "alert sent" field was 0 . When found, the program used the hospital room number stored in the table to access another table with designated alert recipient data (i.e., hospital division, designated computer terminal IP addresses, email addresses, and pager numbers).

Table 1 - Criteria Used by Computer Logic to Identify Patients at High Risk of MRSA Carriage at Admission or During Hospitalization

\footnotetext{
a. Previous positive culture with MRSA from any Intermountain Healthcare

Inpatient or outpatient facility within previous 5 years

b. Current hospital length of stay $\geq 30$ days

c. Patient on hemodialysis

d. Two or more of the following:

1. Age $\geq 75$

2. Acute care hospitalization within previous 6 months

3. Inpatient or outpatient antibiotic (fluoroquinolones, cephalosporins, carbapenems) use within previous 6 months 4. Current hospital length of stay $\geq 10$ days
}

Another Java program designed to "listen" for incoming alerts, was loaded onto all computer terminals designated for alert display. When alerts were received, the "listening" program took control of the computer screen, flashing alternately between a green and black background. The alert content (Figure 2) included the message, "MRSA Alert", the

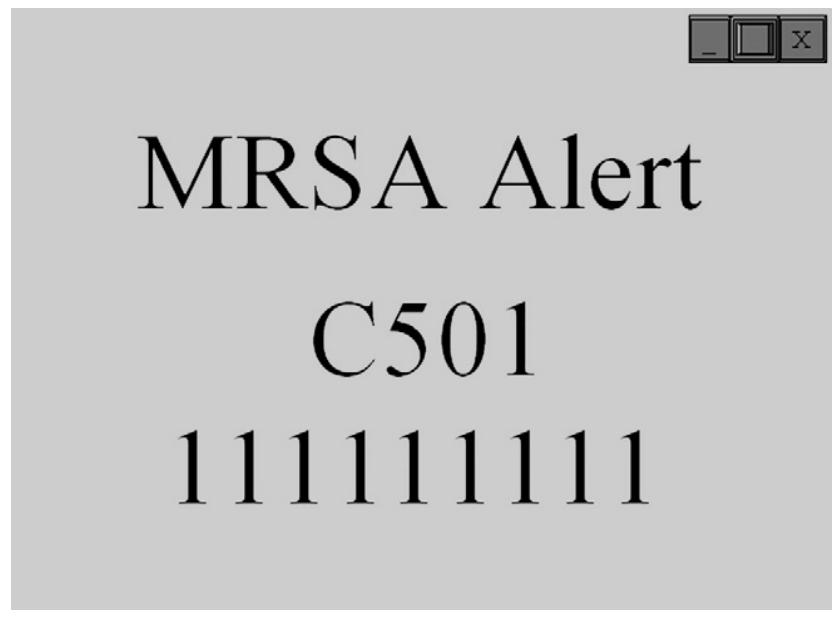

Fig u r e 2. Example of an alert sent to the designated computers on the division of a patient identified at high-risk for MRSA carriage at hospital admission or during hospitalization. Alert contains the room number and the patient encounter number. Nurses acknowledged the alert by clicking on the "close" button in the upper right corner of the screen. 
hospital account number and patient room number. Alert content sent to ICPs and study personnel via e-mail and pagers also included the high-risk criteria and patient name. Clinical end-users stopped the flashing screen by clicking on the "close" button in the upper right corner of the alert window and typing their name in a free-text popup window. The "listening" program then sent a message to the alert server to stop other flashing screens and send a follow-up message to ICPs and study personnel that the alert screen was acknowledged.

\section{Electronic Logic Validation}

The alerting system was tested for 3 months prior to implementation to identify programming errors, network problems or obvious logistical issues. Specific validation outcomes included electronic data capture, rapid high-risk alert deployment, and accurate risk classification. After the MRSA high-risk logic was validated by the programmers, the study coordinator (CJW) made a secondary validation using the electronic medical records of 33 random low risk patients. None of the 33 patients were erroneously identified as low risk. However, $2(6 \%)$ patients had a prior hospitalization or prior antibiotic use outside of the Intermountain Healthcare system documented in a narrative report that was not used by the computer logic.

\section{System Training}

Prior to implementation, study personnel met with the infection control department, hospital executive and management leaders, nursing staff on the participating units, and laboratory staff. Nursing staff recommended: 1) the location of computer terminals for alert display at each of the nursing stations; 2 ) the use of telephone reminders if the nasal swab was not received in the laboratory within 2 hours after alert generation; 3 ) standard nasal swab collection kits would be placed at each nursing station. The decision to place PCR positive patients on contact isolation was made by the ICPs and based on their assessment of the individual patient. Nurses were shown how to acknowledge the terminal alerts and received additional training on the nasal swab collection protocol.

\section{Evaluation Design}

During February and March 2007, MRSA high-risk alerts were generated for patients admitted to or located on three of the five ICUs at LDS Hospital and the nursing unit clinically associated with those ICUs. The three ICUs were randomly selected. In response to a high-risk alert, clinical staff members were instructed to acknowledge the alert and notify nurses to obtain a nasal swab for PCR testing. A verbal and written explanation of the study rationale was provided to all patients prior to nasal swab acquisition. Cost of the laboratory testing was covered by the study and test results were stored into the patient's electronic health record. The study was reviewed and approved as exempt from the informed consent requirement by the University of Utah and the LDS Hospital institutional review boards.

A sample of patients admitted to the study units during February and March 2007 who were identified as low risk patients was also tested for MRSA carriage in order to validate the ability of the alerts to effectively stratify patients on the basis of probability of MRSA carriage. We queried the MRSA Risk database to identify a subset of low risk patients
Table 2 - Results of Computerized MRSA High-risk Alerts and PCR Tests During February and March 2007

\begin{tabular}{|c|c|c|c|}
\hline Category & No. & $\begin{array}{c}\text { PCR } \\
\text { Positive }\end{array}$ & $\begin{array}{c}\% \\
\text { Positive }\end{array}$ \\
\hline $\begin{array}{l}\text { Total high-risk patients identified } \\
\text { Criterion for high risk** }\end{array}$ & 153 & 31 & $20^{*}$ \\
\hline $\begin{array}{l}\text { Previous MRSA infection or } \\
\text { colonization }\end{array}$ & 28 & 17 & 61 \\
\hline Current hospital stay $\geq 30$ days & 11 & 1 & 9.1 \\
\hline Patient on hemodialysis & 31 & 2 & 6.5 \\
\hline $\begin{array}{l}\text { Previous antibiotic and previous } \\
\text { admission }\end{array}$ & 49 & 9 & 18 \\
\hline Previous antibiotic and age $\geq 75$ & 9 & 2 & 22 \\
\hline Previous admission and age $\geq 75$ & 27 & 6 & 22 \\
\hline $\begin{array}{l}\text { Previous antibiotic and } \geq 10 \text { days } \\
\text { hospitalization }\end{array}$ & 6 & 2 & 33 \\
\hline $\begin{array}{l}\text { Age } \geq 75 \text { and } \geq 10 \text { days } \\
\text { hospitalization }\end{array}$ & 16 & 2 & 13 \\
\hline $\begin{array}{l}\text { Previous admission and } \geq 10 \text { days } \\
\text { hospitalization }\end{array}$ & 23 & 4 & 17 \\
\hline
\end{tabular}

${ }^{*} P<0.001$ for total high-risk patients compared to total low-risk patients.

**Patients identified at high risk for MRSA carriage could have met more than one criterion at time of alert.

admitted to the study units within the previous 36 hours on three weekday mornings (Tuesday, Wednesday, and Friday). The low risk patients were approached for study participation by study coordinators using the same protocol as the high-risk patients.

PCR testing was attempted for all patients with high risk alerts during the two-month study period, but only for a systematic sample of half of the low risk patients. This type of sampling is known to introduce "verification bias" into the estimates of sensitivity and specificity, while estimates of positive predictive value and negative predictive value are not affected, ${ }^{23}$ Therefore, we report Begg and Greenes estimates of sensitivity and specificity, where the estimates are corrected for verification bias. ${ }^{24}$ Pepe has shown that the asymptotic confidence interval for these estimates to be unreliable for sample sizes used in research studies and so recommends bootstrapped confidence intervals. ${ }^{23}$ Therefore, we report bootstrapped confidence intervals, using the "bias-corrected and accelerated" method. ${ }^{25}$

\section{Status Report}

During the evaluation period, 31 of 153 (20\%) high-risk patients tested had positive PCR tests compared to 12 of 293 $(4.1 \%)(\mathrm{p}<0.001)$ low risk patients (Table 2). Overall, $20 \%$ of patients were classified as high risk at the time of admission. Among newly admitted patients, the sensitivity of the high risk alerts was $55.9 \%$ (95\% confidence interval: $39.9-73.3 \%)$, specificity $82.4 \%(79.5-85.0 \%)$, positive predictive value $20.3 \%(14.2-27.5 \%)$, and negative predictive value $95.9 \%$ (93.0-97.9\%).

The high-risk patients met 209 of the different high-risk criteria. Some patients had more than one reason to be identified when the alert was generated. Thus, an alert for an admitted patient with previous MRSA and previous antibiotic use plus a previous hospitalization would meet two 
Table 3 - Duration Times for Each Step in the Alerting and Identification of Patients with MRSA Carriage

\begin{tabular}{lccc}
\hline \multicolumn{1}{c}{ Step } & Median (IR) & Mean (95\% CI) & Cumulative mean* (95\% CI) \\
\hline Alert generated on computer screens (Minutes) & $3(1-4)$ & $9(3-15)$ & $9(3-15)$ \\
Alert acknowledged by nurse (Minutes) & $5(2-21)$ & $26(17-35)$ & $36(0.3-116)$ \\
Nasal specimen collection time (Minutes) & $45(13-267)$ & $192(0.2-165)$ & $212(160-264)$ \\
Nasal specimen received in lab (Minutes) & $21(12-38)$ & $37(26-48)$ & $232(2-174)$ \\
MRSA test result available (Hours) & $16.1(10.8-19.4)$ & $15.5(4.7-37.9)$ & $19.2(18.1-20.3)$ \\
\hline
\end{tabular}

$\mathrm{IR}=$ interquartile range, $\mathrm{CI}=$ confidence interval.

${ }^{*}$ Duration of time from when the patient was identified by computer as high-risk for MRSA carriage.

high-risk criteria but only generated one alert. The high-risk criterion with the highest positive predictive value was previous MRSA colonization or infection $(61 \%$, Table 2) followed by previous antibiotic use plus hospitalized $>10$ days $(33 \%)$. Hemodialysis had the lowest positive predictive value $(2$ of $31 ; 6.5 \%)$. Both of the hemodialysis patients with positive PCR tests qualified as high risk on the basis of other criteria (previous MRSA or previous antibiotic use plus previous hospitalization). There were 23 patients identified as high risk based on dialysis alone of which none were MRSA positive. If dialysis was removed as a risk factor, the overall positive predictive value would have been $24 \%$ and if dialysis and the two length of stay risk factors were removed the overall positive predictive value would have been $29 \%$. However, 4 PCR positive patients were identified with the length of stay risk factors (1 of 830 -day only alerts and 3 of 29 10-day alerts).

The cumulative mean interval from MRSA high-risk identification to PCR test requisition was 212 minutes and from PCR test requisition to PCR test result was 15.5 hours (Table 3). Median intervals for individual steps were shorter than mean intervals because of a few instances of prolonged delays. For instance, a network server failure caused the delivery of 7 alerts to be delayed more than 60 minutes and two alerts were delayed 4 and 6 hours. Once the screen alert was activated, we found the average time for the nurse to acknowledge the alert was 26 minutes. We discovered during the study that on some occasions nurses inadvertently hit the "minimize" button in the upper right corner of the alert screen rather than the close button (Figure 2). This resulted in the alert being moved to the task bar on the bottom of the screen and not being closed. The MRSA alert $\log$ stored the time the alert was closed as the acknowledged time. This problem was fixed by making the "minimize" and "maximize" buttons function like the close button. If we found the nasal specimen collection time to be earlier than the alert acknowledge time, we used the specimen time to determine the average for the alert acknowledge step.

Each of the 12 low-risk PCR positive patients were manually checked against the high-risk criteria used for the study and none were found to be high-risk and missed by the computer logic. Two patients did have a previous admission to an Intermountain Healthcare hospital but were before the previous six months. The most common factor found was that 10 of these 12 patients were admitted through the emergency department. The history and physical narrative report for one of those patients documented a previous MRSA pneumonia at a non-Intermountain Healthcare hospital.

\section{Implementation Issues}

A total of 52 implementation issues divided into nine categories were documented (Table 4). Initially, nasal swab collection was delayed on three of four participating units and reminders to staff nurses were required for $56 \%$ of alerts generated during the first 3 weeks of implementation. Problem solving with nurses on these units revealed a lack of urgency for the alert acknowledgment and low prioritization for nasal swab collection. Additional education and workflow improvement provided by the study coordinator

Table 4 - Description and Resolution Status of 52 Implementation Issues Identified During Study

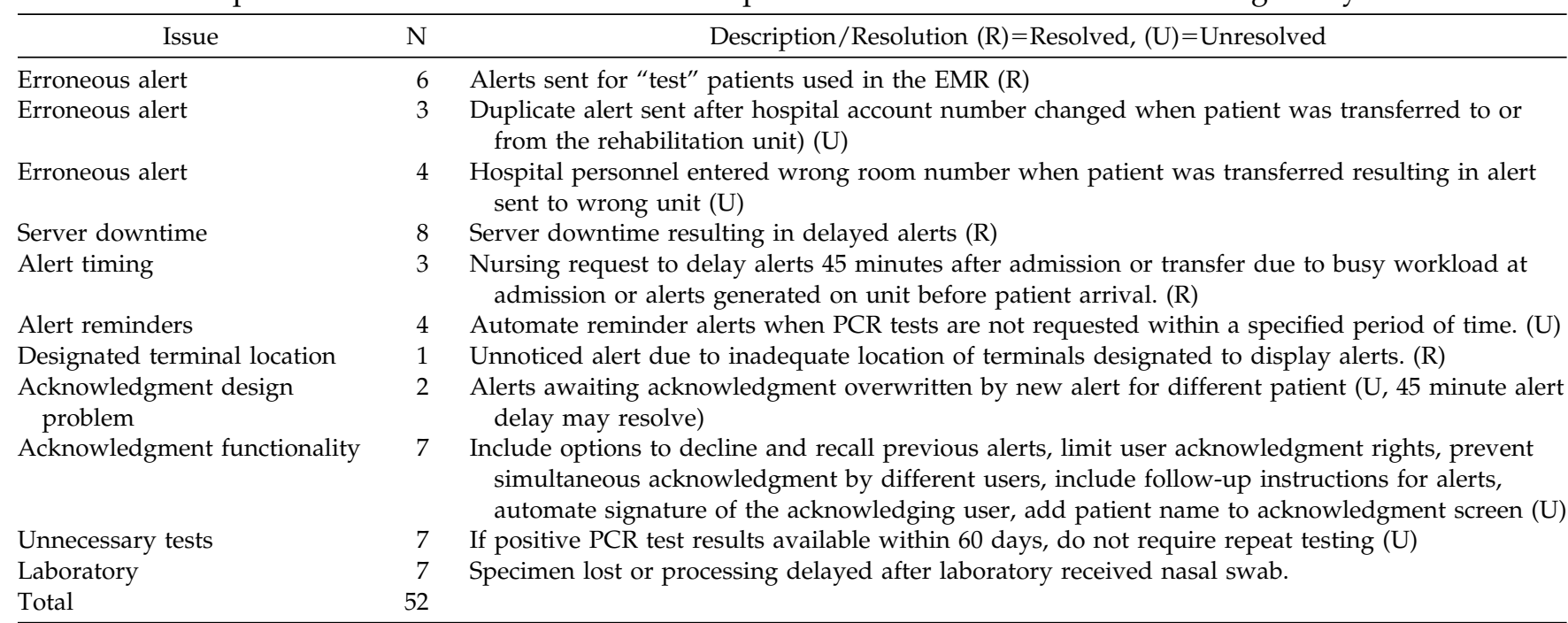


increased the number of alerts not needing reminders and decreased the average nasal swab collection time on the same three units. For the entire study period on all four units, $72 \%$ of the nasal swabs were collected and sent without reminders, $20 \%$ after a reminder call and $8 \%$ were collected and sent by the research staff.

\section{Discussion}

This study demonstrated the feasibility of an innovative approach for targeted MRSA surveillance through the use of computer decision support and access to system-wide healthcare data. It validated the MRSA risk evaluation strategy used in this study and advanced our understanding of implementation requirements and potential logistical issues. Moreover, this study provided an alternative method for the SHEA recommendation to obtain surveillance cultures on all patients on admission. This targeted surveillance strategy also provides a more complete data set to monitor colonization pressure and help improve the identification of hospital acquisition of MRSA. Monitoring colonization pressure has been shown to help reduce MRSA transmission by emphasizing infection control measures. ${ }^{26}$ The plans by Medicare to deny future reimbursement for some hospitalacquired infections demonstrates the financial as well as clinical value of documenting the previously unknown MRSA carriage status of patients. This information will increase the potential to protect critically ill or immunocompromised patients through isolation of identified carriers and improve empiric antibiotic selection. In addition, this study lays the groundwork and provides valuable information for large-scale intervention studies to reduce the transmission of MRSA. A recent study found that computerized reminders for contact isolation based on history of infection with MRSA or VRE improved compliance with infection control recommendations. ${ }^{27}$

These data show that no one risk factor could identify all the patients with MRSA carriage. Thus, if previous MRSA carriage was the only criterion used, 14 (45\%) high-risk patients would have been missed. However, 23 patients were identified as high-risk based on hemodialysis alone and if "hemodialysis" was removed as a risk factor, the positive predictive value would increase from $20.3 \%$ to $24 \%$. Thus, we plan to remove hemodialysis as a risk factor which would decrease the cost and increase the effectiveness by $15 \%$ overall (23 from 153 total alerts) and reduce false positive alerts by $19 \%$ (23 from 122 false positive alerts). We also found that the average total time to identify MRSA carriage was 19.2 hours which should be soon enough to help reduce potential self infection by MRSA or transmission to other patients.

While this study found that computer decision support can help identify patients at high risk for MRSA carriage, that information alone would not have any direct impact on the transmission of MRSA. The information provided by the decision support needs to be applied at the bedside. The only method proven to help reduce MRSA transmission has been the use of intensive infection control methods. The use of contact isolation has been shown to be effective in controlling the spread of MRSA. ${ }^{28,29} \mathrm{New}$ hospital-acquired cases and outbreaks were found to decrease when contact isolation was appropriately used..$^{30}$ The rate of transmission of MRSA from patients on contact isolation was found to be significantly lower than the rate for patients not on isolation. $^{31}$ The fact that not all patients with previous MRSA infection or colonization need to be placed in isolation exemplifies the value of the ICPs to provide a case-by-case analysis of each patient. However, ICPs are not always at the hospital. For this reason the MRSA alerts in this study were also sent to the bedside nurses. Reducing the transmission of MRSA has to be seen as part of all healthcare workers roles.

The use of reminders and the problem solving process improved the timeliness of nasal swab collection in response to alerts. The alert deployment and follow-up process required both technical and systemic problem resolution. As listed in Table 4, the clinicians provided feedback concerning a number of implementation issues and acceptance of the screen alerts. Technical improvements to the alerting system should include automated reminders when PCR test requests are delayed and design changes in the deployment and the timing of the alerts in addition to the acknowledgment process to minimize workflow disruption. Because nurses are busy when patients first arrive, they requested that the MRSA high risk alerts be delayed for 45 minutes. We have been using "flashing screens" to alert for ventilator disconnections and inappropriately programmed infusion pumps for 4 years at LDS Hospital. Clinician acceptance of this alerting method has been high and was requested for the MRSA alerts rather than the use of pagers or email. Based on clinician input, the alert timing was a greater source of annoyance than the flashing screen, however we did receive feedback that it may be wise to reserve the flashing screen for immediate clinical emergencies. Clinicians do not consider the identification of MRSA high-risk status to be an immediate emergency and we plan to test alternate deployment methods in future versions to avoid the potential for alert fatigue.

\section{Limitations}

Although the sample size of this study was limited by the two-month duration, we demonstrated that high-risk patients were 5.2 fold more likely to have a positive PCR test for MRSA than low risk patients. A larger sample size would enable a more precise evaluation of the contribution of individual risk factors or identification of new risk factors to improve the overall predictive value of the system. Also, the high-risk detection algorithm used in this study is dependant on the amount and timing of patient data in the EHR. While the misclassification of patients with a high-risk history is unavoidable if relevant data are missing from the EHR, the results of the high-risk algorithm used in this study could not be duplicated with a system based on human memory and inter-facility communication.

\section{References}

1. Klevens RM, Morrison MA, Nadle J, Petit S, Gershman K, et al. Invasive methicillin-resistant Staphylococcus aureus infections in the United States. JAMA. 2007;298:1763-71.

2. Boyce JM, Cookson B, Christiansen K, Hori S, Vuopio-Varkila J, Kocagöz S, et al. Methicillin-resistant Staphylococcus aureus. Lancet Infect Dis. 2005;10:653-63.

3. Jarvis WR, Schlosser J, Chinn RY, Tweeten S, Jackson M. National prevalence of methicillin-resistant Staphylococcus aureus in inpatients at US health care facilities, 2006. Am J Infect Control. 2007;35:631-7. 
4. National Nosocomial Infections Surveillance System. National Nosocomial Infections Surveillance (NNIS) Systems Report, data summary from January 1992 through June 2004, issued October 2004. Am J Infect Control. 2004;32:470-85.

5. Harbarth S, Martin Y, Rohner P, Henry N, Auckenthaler R, Pittet D. Effect of delayed infection control measures on a hospital outbreak of methicillin-resistant Staphylococcus aureus. J Hosp Infect. 2000;46:43-9.

6. Herwaldt LA. Control of methicillin-resistant Staphylococcus aureus in the hospital setting. Am J Med. 1999;106:11S-18S; discussion $48 \mathrm{~S}-52 \mathrm{~S}$.

7. Richet HM, Mohammed J, McDonald LC, Jarvis WR. Building communication networks: international network for the study and prevention of emerging antimicrobial resistance. Emerg Infect Dis. 2001;7:319-22.

8. Furuno JP, Harris AD, Wright MO, Hartley DM, McGregor JC, Gaff HD, et al. Value of performing active surveillance cultures on intensive care unit discharge for detection of methicillinresistant Staphylococcus aureus. Infect Control Hosp Epidemiol. 2007;28:666-70.

9. Muto CA, Jernigan JA, Ostrowsky BE, Richet HM, Jarvis WR, Boyce JM, Farr BM; SHEA. SHEA guidelines for preventing nosocomial transmission of multidrug-resistant strains of Staphylococcus aureus and enterococcus. Infect Control Hosp Epidermiol. 2003 May; 24(5):362-86.

10. Saint S, Higgins LA, Nallamothu BK, Chenoweth C. Do physicians examine patients in contact isolation less frequently? A brief report. Am J Infect Control. 2003;31:354-6.

11. Stelfox HT, Bates DW, Redelmeier DA. Safety of patients isolated for infection control. JAMA. 2003;290:1899-905.

12. Furuno JP, McGregor JC, Harris AD, Johnson JA, Johnson JK, Langenberg $\mathrm{P}$, et al. Identifying groups at high risk for carriage of antibiotic-resistant bacteria. Arch Intern Med. 2006;166:580-5.

13. Haley CC, Mittal D, Laviolette A, Jannapureddy S, Parvez N, Haley RW. Methicillin-resistant Staphylococcus aureus infection or colonization present at hospital admission: multivariable risk factor screening to increase efficiency of surveillance culturing. J Clin Microbiol. 2007;45:3031-8.

14. Pryor TA, Gardner RM, Clayton PD, Warner HR. The HELP system. J Med Syst. 1983;7:870102.

15. Clayton PD, Narus SP, Huff SM, Pryor TA, Haug PJ, Larkin T, Matney S, Evans RS, Rocha BH, Bowes WA 3rd, Hoston FT, Gundersen ML. Building a comprehensive clinical information system from components. The approach at Intermountain Health Care. Methods Inf Med. 2003;42:1-7.

16. Evans RS, Larsen RA, Burke JP, Gardner RM, Meier FA, Jacobson JA, Conti MT, Jacobson JT, Hulse RK. Computer surveillance of hospital-acquired infections and antibiotic use. JAMA. 1986;256:1007-1011.

17. Pittet D, Safran E, Harbarth S, Borst F, Copin P, Rohner P, Scherrer JR, Auckenthaler R. Automatic alerts for methicillinresistant Staphylococcus aureus surveillance and control: role of a hospital information system. Infect Control Hosp Epidemiol. 1996;17:496-502.
18. Gransden WR, Wingfield J, McConnell G, Branson M. Computerized detection of re-admission of patients with MRSA. J Hosp Infect. 1998;39:160.

19. Primary Children's Medical Center. Salt Lake City, UT, USA. (Personal Communication).

20. Evans RS, Lloyd JF, Abouzelof RH, Taylor CW. System-wide surveillance for clinical encounters by patients previously identified with MRSA and VRE. pp. 212-216 MEDINFO 2004.

21. Evans RS, Johnson KV, Flint VB, Kinder AT, Lyon CR, Hawley WL, Vawdrey DK, Thomsen GE. Enhanced Notification of Critical Ventilator Events. J Am Med Assoc. 2005;12:589-95.

22. Harbarth S, Sax H, Fankhauser-Rodriquez C, Schrenzel J, Agostinho A, Pittet D. Evaluating the probability of previously unknown carriage of MRSA at hospital admission. Am J Med. 2006;119(3):275 e15-23.

23. Pepe MS. The Statistical Evaluation of Medical Tests for Classifcation and Prediction, New York, Oxford University Press, 2003, p. 168-173.

24. Begg CB, Greenes RA. Assessment of diagnostic tests when disease verification is subject to selection bias. Biometrics. 1983;39:207-215.

25. Carpenter J, Bithell J. Bootstrap confidence intervals: when, which, what? A practical guide for medical statisticians. Statist. Med. 2000;19:1141-64.

26. Williams VR, Callery S. Vearncombe M, Simor AE. The role of colonization pressure in nosocomial transmission of methicillinresistant Staphylococcus aureus. Presented at The $34^{\text {th }}$ Annual Education Conference \& International Meeting of the Association for Professionals in Infection Control; June 24-27, 2007; San Jose, Calif.

27. Kho AN, Dexter PR, Warvel JS, Belsito AW, Commiskey M, Wilson SJ, et al. An effective computerized reminder for contact isolation of patients colonized or infected with resistant organisms. Int J Med Inform. 2007;28 [Epub ahead of print].

28. Cooper BS, Stone SP, Kibbler CC, Cookson BD, Roberts JA, Medley GF, Duckworth GJ, Lai R, Ebrahim S. Systematic review of isolation policies in the hospital management of methicillinresistant Staphylococcus aureus: a review of the literature with epidemiological and economic modeling. Health Technol Assess. 2003;7:1-194.

29. Stone SP, Beric V, Quick A, Balestrini AA, Kibbler CC. The effect of an enhanced infection-control policy on the incidence of Clostridium difficile infection and methicillin-resistant Staphylococcus aureus colonization in acute elderly medical patients. Age Ageing. 1998 Sep;27(5):561-8.

30. Hartstein AI, Denny MA, Morthland VH, LeMonte AM, Pfaller MA. Control of methicillin-resistant Staphylococcus aureus in a hospital and an intensive care unit. Infect Control Hosp Epidemiol. 1995 Jul;16(7):405-11.

31. Jernigan JA, Titus MG, Groschel DH, Getchell-White, Farr BM. Effectiveness of contact isolation during a hospital outbreak of methicillin-resistant Staphylococcus aureus. Am J Epidemiol. 1996 Mar 1;143(5):496-504. 